\title{
$A b$ initio study of quantized circular photogalvanic effect in chiral multifold semimetals
}

\author{
Congcong Le, ${ }^{1}$ Yang Zhang $\odot{ }^{2}$ Claudia Felser, ${ }^{1,3}$ and Yan Sun ${ }^{1, *}$ \\ ${ }^{1}$ Max Planck Institute for Chemical Physics of Solids, 01187 Dresden, Germany \\ ${ }^{2}$ Department of Physics, Massachusetts Institute of Technology, Cambridge, Massachusetts 02139, USA \\ ${ }^{3}$ Center for Nanoscale Systems, Faculty of Arts and Sciences, Harvard University, 11 Oxford Street, \\ LISE 308 Cambridge, Massachusetts 02138, USA
}

(Received 28 May 2020; revised 13 July 2020; accepted 8 September 2020; published 30 September 2020)

\begin{abstract}
So far, the circular photogalvanic effect (CPGE) is the only possible quantized signal in Weyl semimetals. With inversion and mirror symmetries broken, Weyl and multifold fermions in band structures with opposite chiralities can stay at different energies and generate a net topological charge. Such a kind of net topological charge can present as a quantized signal in the circular polarized light-induced injection current. According to current theoretical understanding, $\mathrm{RhSi}$ and its counterparts are believed to be the most promising candidates for the experimental observation of the quantized CPGE. However, a real quantized signal has yet to be experimentally observed. Since previous theoretical studies for the quantized CPGE were based on an effective model but not realistic band structures, it should lose some crucial details that influence the quantized signal. The current status motivates us to perform a realistic ab initio study for the CPGE. Our result shows that RhSi and PtAl in chiral multifold semimetals are alternative materials for obtaining the quantized CPGE which is very easy to be interfered with by trivial band-related optic transitions, and a fine tuning of the chemical potential by doping is essential for the observation of the quantized CPGE. We perform an ab initio analysis for the quantized CPGE based on a realistic electronic band structure and provide an effective way to solve the current problem for the given materials.
\end{abstract}

DOI: 10.1103/PhysRevB.102.121111

\section{INTRODUCTION}

Band crossings with a nontrivial topological invariant, including Weyl, Dirac, and unconventional fermions, have been attracting enormous attention in condensed matter [1-3]. For Weyl and Dirac fermions [4-19], they were realized experimentally in topological semimetal materials and further classified into type-I and type-II classes [20]. Unconventional fermions, which contain three-, four-, six-, and eightfold degenerate points, have been exhaustively classified by space group symmetries in solid-state systems with spin-orbit coupling and time-reversal symmetry. These multifold degenerate points with nontrivial topological numbers lead to a series of exotic effects such as surface Fermi arcs [4,5], the chiral anomaly $[21,22]$, large anomalous Hall and spin Hall effects [23-25], and circular photogalvanic effects [26-32].

Recently, it has been pointed out [31-33] that a single multifold degenerate point with a nontrivial invariant can lead to a quantized circular photogalvanic effect (CPGE) trace, which can directly measure the topological charge of degenerate points. So far it is the only quantized signal that exists in topological semimetals. Those topological degenerate points

\footnotetext{
*Corresponding author: ysun@cpfs.mpg.de
}

Published by the American Physical Society under the terms of the Creative Commons Attribution 4.0 International license. Further distribution of this work must maintain attribution to the author(s) and the published article's title, journal citation, and DOI. Open access publication funded by the Max Planck Society. in solid-state systems always obey the Nielsen-Ninomiya theorem as the ground rule [34-36]. A topological degenerate point inevitably accompanies another topological point with opposite charge, because the total charges in the entire Brillouin zone (BZ) must be neutralized. If all degenerate points with opposite charges have the same energy due to crystal symmetries, the total CPGE trace contributed to all degenerate points is zero. In time-reversal and crystal symmetries, only inversion and mirror symmetries can change the charge sign of the degenerate points, indicating that degenerate points with opposite charges have the same energy for systems with inversion and mirror symmetries. Therefore, nonmagnetic chiral topological semimetal materials with a chiral space group, only including time-reversal and rotational symmetries, have different energies for degenerate points with opposite charge and can actually realize a true quantized CPGE trace due to Pauli blocking.

In chiral topological semimetal materials, $\mathrm{RhSi}$ is the most promising candidate to exhibit a quantized CPGE trace. There are two reasons: Multifold degenerate points and exotic Fermi arcs were confirmed by angle-resolved photoemission spectroscopy (ARPES) measurements [37]; and a large energy difference between fourfold and sixfold points exists, which is beneficial to acquire a quantized CPGE trace due to Pauli blocking $[32,33,38]$. Hence, $\mathrm{RhSi}$ has received extensive attention in experiments. However, a theoretical prediction of the quantized CPGE $[32,33]$ has yet to be observed in experiments $[39,40]$. We notice that previous theoretical studies for quantized CPGE are based on few-band effective $k \cdot p$ and tight-binding models by only considering the bands near the degenerate points. Since the 
second-order optical response is very sensitive to the details of the bands, such an approximation easily loses some crucial information that influences the quantized signal. The current situation motivated us to perform an ab initio analysis for the CPGE in $\mathrm{RhSi}$ based on a realistic electronic band structure by first-principles calculations.

This Rapid Communication is organized as follows. In Sec. II, the differences in the CPGE trace between an effective model and real materials are summarized. Then, in Sec. III, a symmetry analysis of the CPGE tensor in the chiral topological semimetal $\mathrm{RhSi}$ is presented. In Sec. IV, we discuss the CPGE trace in $\mathrm{RhSi}$ and other related compounds $(\mathrm{CoSi}$, $\mathrm{PdGa}, \mathrm{PtGa}, \mathrm{PtAl}, \mathrm{RhSn}$ ). Finally, in Sec. V, we give a summary.

\section{DIFFERENCE BETWEEN EFFECTIVE MODEL AND REAL MATERIALS}

From the effective models for multifold degenerate points with a linear dispersion, the exact quantization of the CPGE trace is predicted in a certain frequency range $[31,32]$. The following is a brief summary of the derivation of a quantized CPGE trace [31,32]. The CPGE trace can be rewritten as

$$
\beta(\omega)=\frac{4 \pi^{2} \beta_{0}}{V} \sum_{\vec{k}, i, n, m} f_{n m}^{\vec{k}} \Delta_{\vec{k}, n m}^{i} R_{\vec{k}, n m}^{i} \delta\left(\hbar \omega-E_{\vec{k}, m n}\right),
$$

where $\beta_{0}=\frac{\pi e^{3}}{h^{2}}$, and the relation between $\vec{R}_{\vec{k}, n m}$ and the Berry curvature is $\vec{\Omega}_{\vec{k}, n}=i \sum_{m \neq n} \vec{R}_{\vec{k}, n m}$.

The topological property of the degenerate points can be characterized by the Chern number,

$$
\mathcal{C}_{n}=\frac{1}{2 \pi} \oint_{S} \vec{\Omega}_{n}(\vec{k}) \cdot d \vec{S}_{n}
$$

where $\vec{S}_{n}$ is a closed surface of band $n$ enclosing the degenerate points, and $\vec{\Omega}_{n}(\vec{k})=\nabla_{\vec{k}} \times\left\langle\psi_{n}(\vec{k})\left|i \nabla_{\vec{k}}\right| \psi_{n}(\vec{k})\right\rangle$ is the Berry curvature of band $n$.

In spherical coordinates, the CPGE trace becomes

$$
\begin{aligned}
\beta(\omega) & =4 \pi^{2} \beta_{0} \sum_{k, n, m} \int \frac{k^{2} d k d \Omega}{(2 \pi)^{3}} \partial_{\vec{k}} E_{\vec{k}, n m} \cdot \vec{R}_{\vec{k}, n m} \delta\left(\hbar \omega-E_{\vec{k}, m n}\right) \\
& =4 \pi^{2} \beta_{0} \sum_{n, m} \int \frac{k^{2} d k d \Omega}{(2 \pi)^{3}} \partial_{k} E_{k, n m}^{\hat{k}} R_{k, n m}^{\hat{k}} \delta\left(\hbar \omega-E_{\vec{k}, m n}\right) \\
& =4 \pi^{2} \beta_{0} \sum_{n, m} \int \frac{d\left(E_{k, n m}\right) d \Omega}{(2 \pi)^{3}} k^{2} R_{k, n m}^{\hat{k}} \delta\left(\hbar \omega-E_{\vec{k}, m n}\right) \\
& =4 \pi^{2} \beta_{0} \sum_{n, m} \int \frac{d \Omega}{(2 \pi)^{3}} k^{2}(\hbar \omega) R_{n m}^{\hat{k}}(\hbar \omega) \\
& =4 \pi^{2} \beta_{0} \sum_{n, m} \int d S_{n m}^{\hat{k}} R_{n m}^{\hat{k}}, \\
& =4 \pi^{2} \beta_{0} \sum_{n, m} \int d \vec{S}_{n m} \cdot \vec{R}_{n m},
\end{aligned}
$$

where $\partial_{\vec{k}} E_{\vec{k}, n m}=\partial_{k} E_{k, n m}^{\hat{k}} \hat{k}+\frac{1}{k} \partial_{\theta} E_{k, n m}^{\hat{\theta}} \hat{\theta}+\frac{1}{k \sin \theta} \partial_{\phi} E_{k, n m}^{\hat{\phi}} \hat{\phi}$ and $\vec{R}_{\vec{k}, n m}=R_{k, n m}^{\hat{k}} \hat{k}+R_{k, n m}^{\hat{\theta}} \hat{\theta}+R_{k, n m}^{\hat{\phi}} \hat{\phi}$, and $\vec{R}_{n m}$ has only a radial component in the spherical coordinates for multifold degenerate points with a linear dispersion [32]. For a given frequency $\omega$, the delta function and Fermi-Dirac distributions select a surface $\vec{S}_{n m}$ in the $\vec{k}$ space where $E_{\vec{k}, n m}=\hbar \omega$, and $d \vec{S}$ denotes the oriented surface element normal to $\vec{S}$. Hence the CPGE trace is physically understood as the Berry flux penetrating through $\vec{S}$. For a single type-I Weyl point, when the Fermi level is located at the Weyl point, the CPGE trace is $\beta(\omega)=$ $i C \beta_{0}$, where $C$ is the Chern number of the occupied band. Similar to type-I Weyl points, other multifold fermions can also obtain a quantized CPGE trace according to the above derivation.

The above results are based on the effective $k \cdot p$ models of multifold fermions. However, for real nonmagnetic chiral topological semimetal materials, there are some factors that affect the quantized CPGE trace:

(1) Except for the bands that form the degenerate points, there are many extra bands in real materials, which can induce corrections of the relation between $\vec{R}$ and $\vec{\Omega}_{n}$ compared with that of the effective model.

(2) The condition for quantization is that the band structures near the multifold fermion have a linear dispersion, indicating that higher-order band dispersions can modify the CPGE trace.

(3) The nontrivial band structures that form multifold fermions can contribute a quantized CPGE trace, while other trivial band structures are allowed optical transitions and can provide a CPGE.

\section{SYMMETRY ANALYSIS OF CPGE TENSOR}

The crystal structure of RhSi has a nonsymmorphic space group $G=P 2{ }_{1} 3$ (No. 198), and the quotient group $G / T$ is specified by 12 symmetry operations, where we denote $T$ as the translation group with respect to the unit cell. In 16 symmetry operations, the nonsymmorphic symmetry operations are $\tilde{C}_{2 x}=\left\{C_{2 x} \mid 1 / 2,1 / 2,0\right\}, \tilde{C}_{2 y}=\left\{C_{2 y} \mid 0,1 / 2,1 / 2\right\}$, and $\tilde{C}_{2 z}=\left\{C_{2 y} \mid 1 / 2,0,1 / 2\right\}$. Under crystal symmetries $g_{\tau}=$ $\tilde{C}_{2 x / y / z}$, the relations of $\tilde{\beta}_{i j}(\vec{k}, \omega)$ and $\tilde{\beta}_{i j}(g \vec{k}, \omega)$ can be written as

$$
\begin{aligned}
& \tilde{\beta}_{i i}(g \vec{k}, \omega)=\tilde{\beta}_{i i}(\vec{k}, \omega) \quad(i=j), \\
& \tilde{\beta}_{i j}(g \vec{k}, \omega)=-\tilde{\beta}_{i j}(\vec{k}, \omega) \quad(i \neq j) .
\end{aligned}
$$

Due to $\tilde{C}_{2 x / y / z}$ rotational symmetries, $\beta_{i j}(\omega)(i \neq j)$ is zero and $\beta_{i i}(\omega)$ is nonzero. Under the crystal symmetry $g=C_{3,111}$, the relations of $\tilde{\beta}_{i j}(\vec{k}, \omega)$ and $\tilde{\beta}_{i j}(g \vec{k}, \omega)$ can be given by

$$
\begin{aligned}
\tilde{\beta}_{x x}(\vec{k}, \omega) & =\tilde{\beta}_{z z}\left(C_{3,111} \vec{k}, \omega\right), \quad \tilde{\beta}_{y y}(\vec{k}, \omega)=\tilde{\beta}_{x x}\left(C_{3,111} \vec{k}, \omega\right), \\
\tilde{\beta}_{z z}(\vec{k}, \omega) & =\tilde{\beta}_{y y}\left(C_{3,111} \vec{k}, \omega\right) .
\end{aligned}
$$

The above derivation details are given in the Supplemental Material [41]. In the calculation below, we will focus on the diagonal CPGE tensor $\beta_{i i}(\omega)$.

\section{AB INITIO ANALYSIS OF CIRCULAR PHOTOGALVANIC EFFECT (CPGE)}

To calculate the CPGE tensor, we project the $a b$ initio DFT Bloch wave function into high symmetric atomic-orbital-like Wannier functions [42] with a diagonal position operator, 

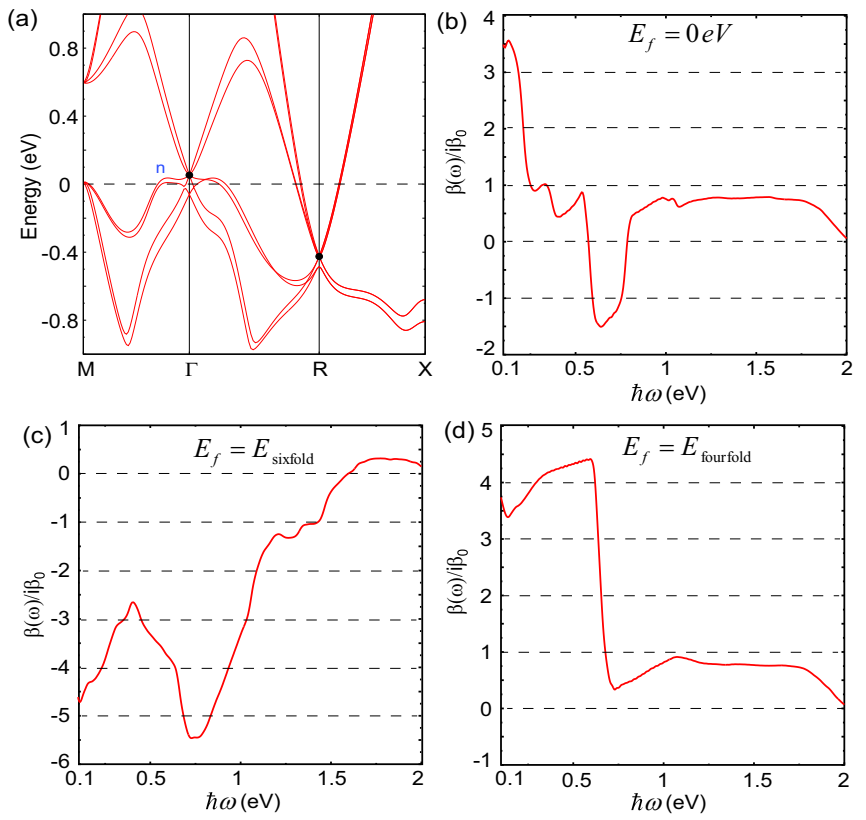

FIG. 1. (a) Band structure of RhSi with SOC in the paramagnetic state; black points denote fourfold and sixfold degenerate points at the $\Gamma$ and $R$ points, and the numbers $n$ with a blue color represent a band number of four degenerate points. (b)-(d) show the trace of the CPGE tensor as a function of frequency $\hbar \omega$ by density functional theory (DFT) calculations with a corresponding Fermi level $E_{f}=$ $0 \mathrm{eV}, E_{\text {sixfold }}$, and $E_{\text {fourfold }}$, respectively.

as performed in the code of the full-potential local-orbital (FPLO) minimum basis $[43,44]$. To obtain precise Wannier functions, we include the outermost $d, s$, and $p$ orbitals for $\mathrm{Rh}$, and the outermost $s$ and $p$ orbitals for the element $\mathrm{Si}$, which guarantees a full band overlap from the $a b$ initio and Wannier functions in the energy window from -10 to $10 \mathrm{eV}$. With highly symmetric Wannier functions, we construct an effective tight-binding model Hamiltonian and calculate the photoconductivity by Eq. (1) in the Supplemental Material [41], where we use a diagonal tight-binding approximation $[45,46]$. The influence of the off-diagonal components of the position matrix has been well studied [45,46], and the contribution from an off-diagonal part may come from the derivation of $\partial_{b} r_{k n m}^{a}$. The tensor $\beta$ of CPGE does not include the derivation term, indicating that the changes from the offdiagonal component are quite small. What is more, in this Rapid Communication, the CPGE trace in the RhSi results from trivial and nontrivial band structures, where nontrivial band structures form multifold fermions. We focus on the quantization of the CPGE trace caused by nontrivial band structures near the multifold nodes, and the topological invariant of the multifold nodes determines the CPGE trace. Since topological invariants are not affected by perturbations, the off-diagonal position matrix elements do not change the topological invariant of the multifold nodes, suggesting that the diagonal tight-binding approximation is valid for the quantization of the CPGE trace caused by multifold nodes.

Figure 1(a) shows the band structure of $\mathrm{RhSi}$ with spinorbit coupling (SOC) in the paramagnetic state. Near the Fermi level, the valence and conduction bands are mainly (a)
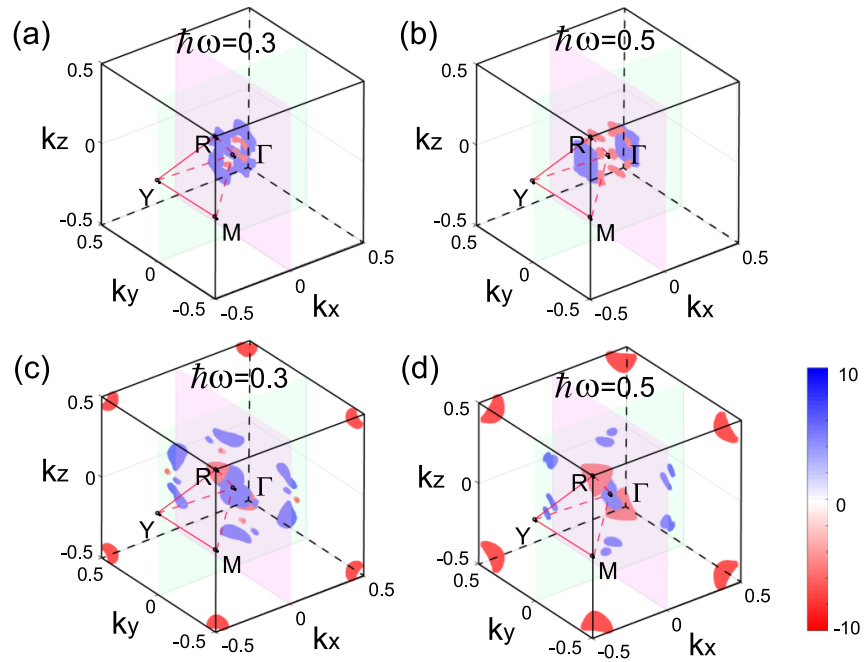

FIG. 2. (a), (b) Momentum distribution of the CPGE tensor $\tilde{\beta}_{x x}$ from all bands without adjusting the Fermi level at frequencies $\hbar \omega=0.3$ and $0.5 \mathrm{eV}$. (c), (d) Momentum distribution of the CPGE tensor $\tilde{\beta}_{x x}$ in the BZ from all bands with a Fermi level $E_{f}=E_{\text {sixfold }}$ at frequencies $\hbar \omega=0.3$ and $0.5 \mathrm{eV}$. We control the range $[-10,10]$ of the color bar in MATLAB, which is equivalent to being normalized by a constant.

attributed to the Rh- $4 d$ orbitals. A fourfold degeneracy at the $\Gamma$ points with an energy $E_{\text {fourfold }}=50 \mathrm{meV}$ is protected by point group $T$ and time-reversal symmetry, which can be understood by the character tables of point group $T$. A sixfold degeneracy at the $R$ points with an energy $E_{\text {sixfold }}=-400 \mathrm{meV}$ is protected by nonsymmorphic symmetries $\left(\left\{C_{3,111}^{-1} \mid 010\right\},\left\{C_{2 x} \mid \frac{1}{2} \frac{3}{2} 0\right\}\right.$, and $\left.\left\{C_{2 y} \mid 0 \frac{3}{2} \frac{31}{2}\right\}\right)$ and time-reversal symmetry [3]. Because the degeneracy points at $\Gamma$ and $R$ are not symmetrically related, the energies of these points can be different. The fourfold degeneracy at the $\Gamma$ point is described as a spin-3/2 fermion with a Chern number $C=3,1,-1,-3$ for four bands [33,38], and the sixfold degeneracy at the $R$ point is a double spin- 1 fermion with $C=2,2,0,0,-2,-2$ for six bands, which satisfies the Nielsen-Ninomiya theorem [34-36].

We first calculated the trace of the CPGE tensor with the Fermi level lying at the charge neutral points, which should be close to the case in experimental measurements [39]. As presented in Fig. 1(b), one cannot see the quantized value in the long range of frequency from 0.1 to $2.0 \mathrm{eV}$, and the plateau close to $4 \beta_{0}$ is absent, which is consistent with experimental results [39]. To explain that the quantization disappears in Fig. 1(b), we take two frequencies as examples to calculate the local momentum distribution of the CPGE tensor $\tilde{\beta}_{x x}$. When two degenerate points with opposite charge can simultaneously provide the CPGE, the quantization will disappear and the total of the CPGE is close to zero. Here, two frequencies $\hbar \omega=0.3$ and $0.5 \mathrm{eV}$ are chosen, where the fourfold degenerate point at the $\Gamma$ point can contribute the CPGE and the sixfold degenerate point at the $R$ point is forbidden due to Pauli blocking, indicating that optical transition nontrivial bands only come from four bands that make up the fourfold degeneracy point near the $\Gamma$ point. From the momentum distribution of the CPGE tensor $\tilde{\beta}_{x x}$ in the BZ from all bands 
(a)

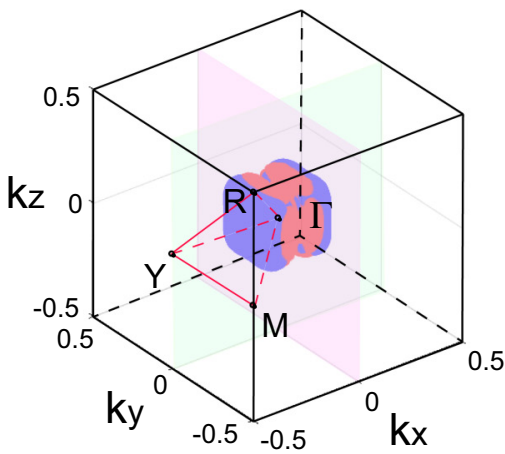

(b)

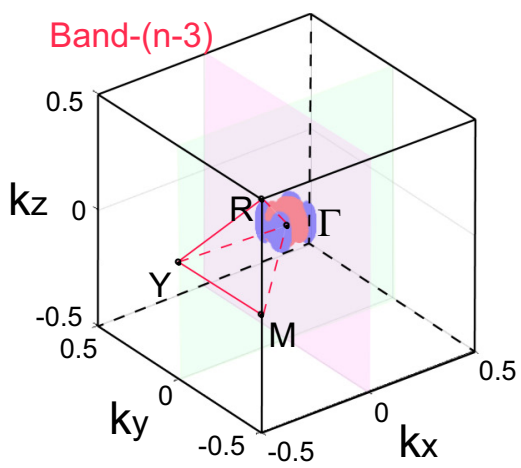

(c)

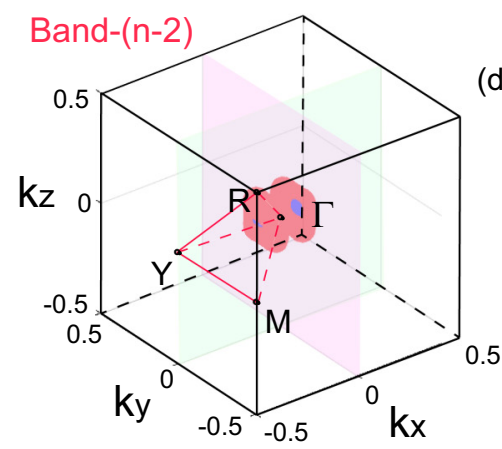

(d)

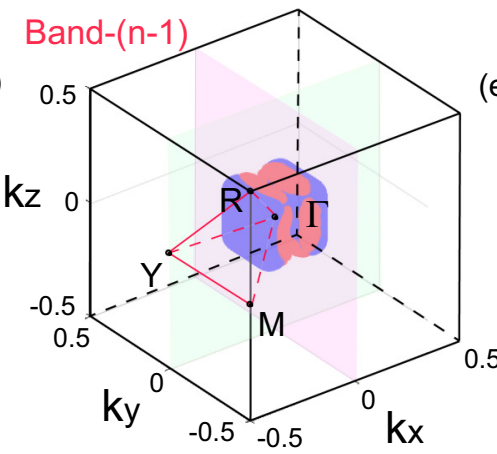

(e)

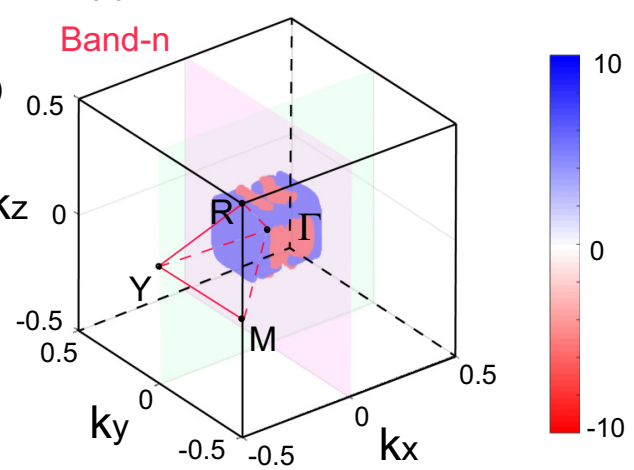

FIG. 3. Momentum distribution of the CPGE tensor $\tilde{\beta}_{x x}$ in the $\mathrm{BZ}$ at frequency $\hbar \omega=0.5 \mathrm{eV}$ with a corresponding Fermi level $E_{f}=E_{\text {fourfold }}$. (a) Momentum distribution from all bands with optically allowed transitions. (b)-(e) Momentum distribution from band ( $n-3)$ to band $n$, respectively. We control the range $[-10,10]$ of the color bar in MATLAB, which is equivalent to being normalized by a constant.

at frequencies $\hbar \omega=0.3$ and $0.5 \mathrm{eV}$ in Figs. 2(a) and 2(b), one can see that $k$ points far away from the $\Gamma$ point greatly contribute to the CPGE, and do not form a closed surface in the BZ. Hence, the trivial bands are mainly attributed to the CPGE trace and the closed surface $\vec{S}$ wrapping degenerate point is also absent. As discussed in Sec. II, the single multifold degenerate point with a nontrivial topological charge can contribute to the quantized CPGE trace in a certain frequency range, and the CPGE trace can be understood as the Berry curvature penetrating through the closed surface $\vec{S}$ wrapping degenerate point. The results in Figs. 2(a) and 2(b) do not satisfy the conditions for obtaining a quantized CPGE trace, and hence the quantization of the CPGE trace with the Fermi level lying at the charge neutral point cannot be observed in our calculations.

To further explore the quantized CPGE trace in RhSi and provide clues for experimental research, we can dope the holes and electrons in our calculations by reducing and raising the Fermi level. In doping holes the quantized CPGE trace is always absent, while the quantized platform close to $4 \beta_{0}$ can be acquired in a certain range in doping electrons. In the following, we will describe both cases in detail.

In doping holes in $\mathrm{RhSi}$ by reducing the Fermi level, the quantized platform is always absent in a certain range of doping holes. We will analyze the reasons by focusing on the case of a Fermi level at sixfold degeneracy with a corresponding 1.363 hole doping per unit cell, where the optical transitions near the fourfold fermion are forbidden due to Pauli blocking in the frequency range from 0.1 to $0.5 \mathrm{eV}$. Figure 1(c) shows the CPGE trace with a Fermi level $E_{f}=E_{\text {sixfold }}$, and the plateau close to $4 \beta_{0}$ is absent. Figures 2(c) and 2(d) show a momentum distribution of the CPGE tensor $\tilde{\beta}_{x x}$ in the BZ from all bands at frequencies $\hbar \omega=0.3$ and $0.5 \mathrm{eV}$, and the $k$ points near the $R$ point and other places in the $\mathrm{BZ}$ can contribute to CPGE. Hence, in addition to nontrivial bands that form a sixfold fermion at the $R$ point contributing to CPGE, other trivial bands have also contributed significantly to CPGE. What is more, the CPGE trace provided by trivial bands can compete with that contributed by nontrivial bands, indicating that the quantization of the CPGE trace disappears.

In doping electrons, we focus on the Fermi level at fourfold degeneracy with a corresponding 0.067 electron doping per unit cell. A nearly quantized platform close to $4 \beta_{0}$ with a frequency from 0.1 to $0.6 \mathrm{eV}$ is shown in Fig. 1(d), where in the frequency range the optical transitions near $\Gamma$ can contribute to the CPGE trace and the nontrivial bands that form a fourfold fermion play a major role. When the optical frequency $\omega$ is more than $0.6 \mathrm{eV}$, the nontrivial bands from a sixfold fermion can begin to provide a CPGE trace, and cancel the contribution from the fourfold fermion, indicating that the CPGE trace is close to zero from 0.7 to $2 \mathrm{eV}$. As discussed in Sec. II, some contributions to CPGE from other trivial bands cannot be ruled out, and the exact quantization $4 \beta_{0}$ range from 0.1 to $0.6 \mathrm{eV}$ and exact zero range from 0.6 to $2 \mathrm{eV}$ are absent in the DFT calculations. To analyze the CPGE trace further, we calculate the momentum distribution of the CPGE trace in the BZ in the above different frequency ranges. First, Fig. 3 shows the momentum distribution of the CPGE trace at a frequency $\hbar \omega=0.5 \mathrm{eV}$. Figure 3(a) displays momentum distributions from all bands with optically allowed transitions, indicating that the CPGE completely contributes to the electric optical transitions of bands near $\Gamma$. Momentum distributions from the band $(n-3)$ to band $n$ are 

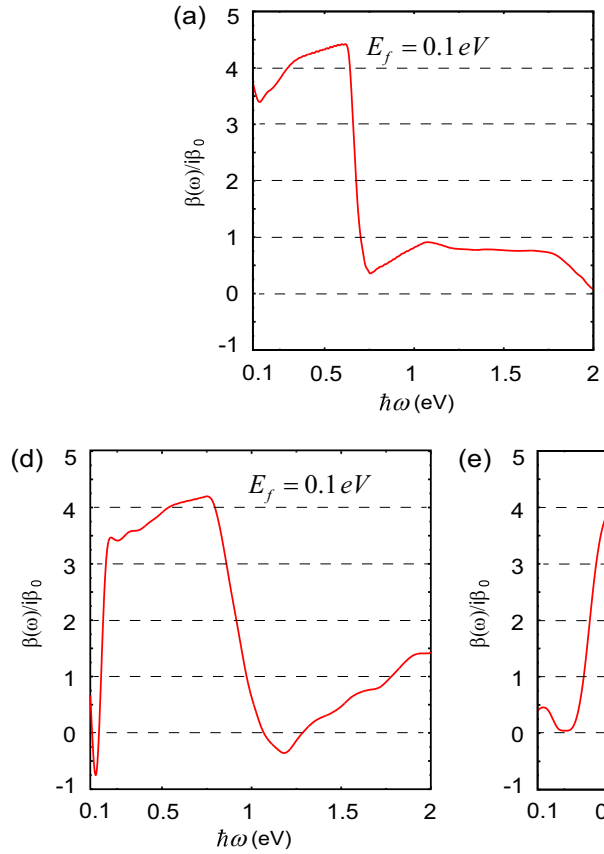
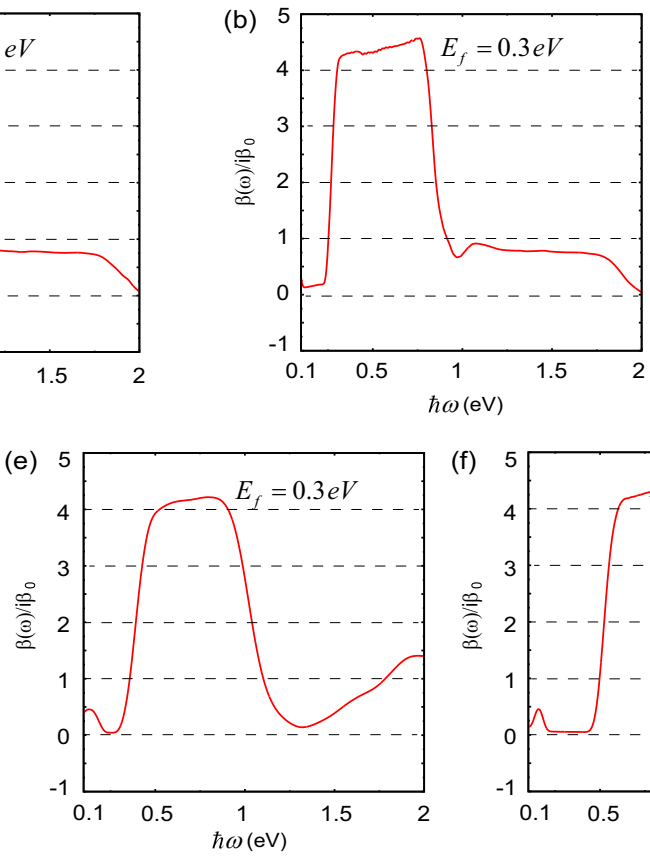
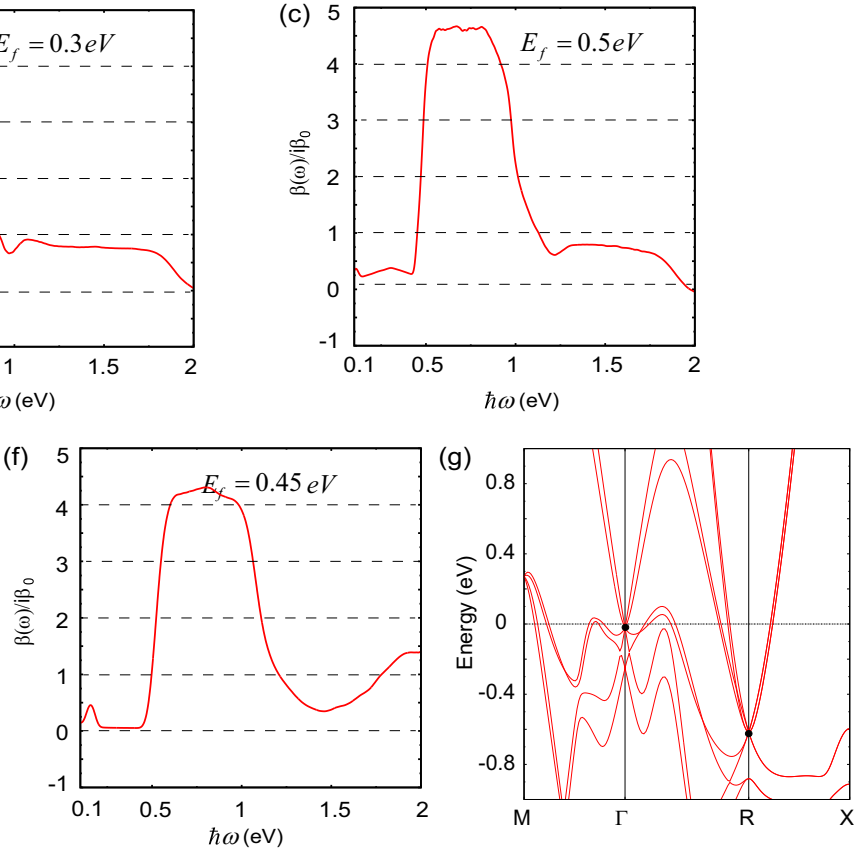

FIG. 4. The trace of the CPGE tensor as a function of frequency $\hbar \omega$ by DFT calculations with different Fermi levels: (a)-(c) RhSi and (d)-(f) PtAl. (g) Band structure of PtAl with SOC in the paramagnetic state; the black points denote fourfold and sixfold degenerate points at the $\Gamma$ and $R$ points.

shown in Figs. 3(b)-3(e), respectively. The nontrivial band $(n-2)$ and $n$ from a fourfold fermion are mainly attributed to the CPGE trace, and the trivial band $(n-3)$ and $n-1$ can provide a small but finite CPGE trace, suggesting that the exact quantization $4 \beta_{0}$ is absent. Second, Figs. S1(a)S1(f) in the Supplemental Material [41] show momentum distributions of the CPGE trace in the BZ from all bands with optically allowed transitions, where the frequency range is from $\hbar \omega=0.7 \mathrm{eV}$ to $\hbar \omega=1.8 \mathrm{eV}$. In the range from 0.7 to $1.1 \mathrm{eV}$, the CPGE trace mainly contributes to the electric optical transitions near the $\Gamma$ and $R$ points shown in Figs. S1(a)-S1(c). Figures S1(d)-S1(f) show the CPGE trace of frequency from 1.2 to $1.8 \mathrm{eV}$, which is provided from other places in the BZ. Hence, an exact zero of a frequency from 0.6 to $2 \mathrm{eV}$ is absent and is close to zero.

Except for the Fermi level at fourfold degeneracy in the doping electrons, we also calculate the trace of the CPGE tensor at other Fermi levels. In the Fermi level from 0.1 to $0.5 \mathrm{eV}$, a large quantized platform can be obtained, and slowly disappears when the Fermi level is greater than $0.5 \mathrm{eV}$. Figures 4(a)-4(c) show the trace of the CPGE tensor as a function of frequency $\hbar \omega$, where the Fermi levels are 0.1, 0.3 , and $0.5 \mathrm{eV}$ with corresponding $0.089,0.185$, and 0.360 electron doping per unit cell. The quantized platform close to $4 \beta_{0}$ always exists in a large frequency range, where the nontrivial bands from the fourfold degenerate point at the $\Gamma$ point can contribute CPGE, and the sixfold degenerate point at the $R$ point is forbidden due to Pauli blocking. Hence, these results open up more possibilities for experimentally observed quantized CPGE traces in $\mathrm{RhSi}$.

Since there are other related compounds $(\mathrm{CoSi}, \mathrm{PdGa}$, $\mathrm{PtGa}, \mathrm{PtAl}, \mathrm{RhSn}$ ) similar to RhSi in the experiment, we also calculate the CPGE trace of these compounds. Among these compounds, $\mathrm{PtAl}$ is also an alternative material for obtaining a quantized CPGE. Figure 4(g) shows the band structure of PtAl with SOC in the paramagnetic state, where the fourfold and sixfold degenerate points denoted by black points are close to and away from the Fermi level, respectively. In our calculations, the quantized CPGE trace is always absent in doping holes, and the quantized platform close to $4 \beta_{0}$ can be acquired in a certain frequency range in doping electrons. In doping electrons, in a Fermi level from 0.1 to $0.45 \mathrm{eV}$, a large quantized platform can be obtained. When the Fermi levels are set at $0.1,0.3$, and $0.45 \mathrm{eV}$ with corresponding $0.134,0.252$, and 0.332 electron doping per unit cell, a quantized CPGE trace can be obtained, as shown in Figs. 4(d)-4(f). Hence, PtAl has the great potential to obtain a quantized platform in doping electrons.

\section{CONCLUSION}

In summary, the circular photogalvanic effect (CPGE) of topological semimetals with chiral multifold fermions was calculated based on realistic ab initio band structures. We find that the quantized signal is very sensitive to the trivial bands away from the high symmetry points. A nearly quantized value equal to the topological charge of chiral multifold fermions can be obtained by tiny doping. When the chemical potential is around from 0.1 to $0.5 \mathrm{eV}$, a plateau close to 4 exists in the frequency range. In addition to $\mathrm{RhSi}, \mathrm{PtAl}$ is also an alternative material for obtaining quantized CPGE, which appears in a certain range.

Note added. Recently, we became aware of an experimental study on the same material with a fully $a b$ initio calculation [40]. 


\section{ACKNOWLEDGMENTS}

We thank Dennis Nenno for helpful discussions. This work was financially supported by the ERC Advanced Grant No. 291472 Idea Heusler, ERC Advanced Grant No. 742068 TOPMAT. This work was performed in part at the Center for
Nanoscale Systems (CNS), a member of the National Nanotechnology Coordinated Infrastructure Network (NNCI), which is supported by the National Science Foundation under NSF Award no.1541959. CNS is part of Harvard University. Some of our calculations were carried out on the Cobra cluster of MPCDF, Max Planck society.
[1] C.-K. Chiu, J. C. Y. Teo, A. P. Schnyder, and S. Ryu, Rev. Mod. Phys. 88, 035005 (2016).

[2] N. P. Armitage, E. J. Mele, and A. Vishwanath, Rev. Mod. Phys. 90, 015001 (2018).

[3] B. Bradlyn, J. Cano, Z. Wang, M. G. Vergniory, C. Felser, R. J. Cava, and B. A. Bernevig, Science 353, aaf5037 (2016).

[4] G. Xu, H. M. Weng, Z. J. Wang, X. Dai, and Z. Fang, Phys. Rev. Lett. 107, 186806 (2011).

[5] X. G. Wan, A. M. Turner, A. Vishwanath, and S. Y. Savrasov, Phys. Rev. B 83, 205101 (2011).

[6] S. Murakami, New J. Phys. 9, 356 (2007).

[7] A. A. Burkov and L. Balents, Phys. Rev. Lett. 107, 127205 (2011).

[8] S.-Y. Xu, I. Belopolski, N. Alidoust, M. Neupane, G. Bian, C. Zhang, R. Sankar, G. Chang, Z. Yuan, C.-C. Lee, S.-M. Huang, H. Zheng, J. Ma, D. S. Sanchez, B. Wang, A. Bansil, F. Chou, P. P. Shibayev, H. Lin, S. Jia, and M. Z. Hasan, Science 349, 613 (2015)

[9] B. Q. Lv, N. Xu, H. Weng, J. Ma, P. Richard, X. Huang, L. Zhao, G. Chen, C. Matt, F. Bisti, V. Strocov, J. Mesot, Z. Fang, X. Dai, T. Qian, M. Shi, and H. Ding, Nat. Phys. 11, 724 (2015).

[10] B. Q. Lv, H. M. Weng, B. B. Fu, X. P. Wang, H. Miao, J. Ma, P. Richard, X. C. Huang, L. X. Zhao, G. F. Chen, Z. Fang, X. Dai, T. Qian, and H. Ding, Phys. Rev. X 5, 031013 (2015).

[11] C. Shekhar, A. K. Nayak, Y. Sun, M. Schmidt, M. Nicklas, I. Leermakers, U. Zeitler, Y. Skourski, J. Wosnitza, Z. Liu, Y. Chen, W. Schnelle, H. Borrmann, Y. Grin, C. Felser, and B. Yan, Nat. Phys. 11, 645 (2015).

[12] L. X. Yang, Z. K. Liu, Y. Sun, H. Peng, H. F. Yang, T. Zhang, B. Zhou, Y. Zhang, Y. F. Guo, M. Rahn, D. Prabhakaran, Z. Hussain, S.-K. Mo, C. Felser, B. Yan, and Y. L. Chen, Nat. Phys. 11, 728 (2015).

[13] F. de Juan, Y. Zhang, T. Morimoto, Y. Sun, J. E. Moore, and A. G. Grushin, Phys. Rev. Research 2, 012017(R) (2020).

[14] S.-Y. Xu, N. Alidoust, I. Belopolski, Z. Yuan, G. Bian, T.-R. Chang, H. Zheng, V. N. Strocov, D. S. Sanchez, G. Chang, C. Zhang, D. Mou, Y. Wu, L. Huang, C.-C. Lee, S.-M. Huang, B. Wang, A. Bansil, H.-T. Jeng, T. Neupert et al., Nat. Phys. 11, 748 (2015).

[15] S. M. Young, S. Zaheer, J. C. Y. Teo, C. L. Kane, E. J. Mele, and A. M. Rappe, Phys. Rev. Lett. 108, 140405 (2012).

[16] Z. J. Wang, Y. Sun, X. Q. Chen, C. Franchini, G. Xu, H. M. Weng, X. Dai, and Z. Fang, Phys. Rev. B 85, 195320 (2012).

[17] Z. J. Wang, H. M. Weng, Q. S. Wu, X. Dai, and Z. Fang, Phys. Rev. B 88, 125427 (2013).

[18] C. Le, S. Qin, X. Wu, X. Dai, P. Fu, C. Fang, and J. Hu, Phys. Rev. B 96, 115121 (2017).

[19] C. Le, X. Wu, S. Qin, Y. Li, R. Thomale, F.-C. Zhang, and J. $\mathrm{Hu}$, Proc. Natl. Acad. Sci. USA 115, 8311 (2018).

[20] A. A. Soluyanov, D. Gresch, Z. J. Wang, Q. S. Wu, M. Troyer, X. Dai, and B. A. Bernevig, Nature (London) 527, 495 (2015).

[21] A. A. Burkov, J. Phys.: Condens. Matter 27, 113201 (2015).
[22] E. V. Gorbar, V. A. Miransky, and I. A. Shovkovy, Phys. Rev. B 89, 085126 (2014).

[23] E. Liu, Y. Sun, N. Kumar, L. Muechler, A. Sun, L. Jiao, S.-Y. Yang, D. Liu, A. Liang, Q. Xu et al., Nat. Phys. 14, 1125 (2018)

[24] Q. Wang, Y. Xu, R. Lou, Z. Liu, M. Li, Y. Huang, D. Shen, H. Weng, S. Wang, and H. Lei, Nat. Commun. 9, 3681 (2018).

[25] Y. Sun, Y. Zhang, C. Felser, and B. Yan, Phys. Rev. Lett. 117, 146403 (2016).

[26] S. Zhong, J. E. Moore, and I. Souza, Phys. Rev. Lett. 116, 077201 (2016).

[27] J. Ma and D. A. Pesin, Phys. Rev. B 92, 235205 (2015).

[28] C.-K. Chan, N. H. Lindner, G. Refael, and P. A. Lee, Phys. Rev. B 95, 041104(R) (2017).

[29] Q. Ma, S.-Y. Xu, C.-K. Chan, C.-L. Zhang, G. Chang, Y. Lin, W. Xie, T. Palacios, H. Lin, S. Jia, P. A. Lee, P. Jarillo-Herrero, and N. Gedik, Nat. Phys. 13, 842 (2017).

[30] J. E. Sipe and A. I. Shkrebtii, Phys. Rev. B 61, 5337 (2000).

[31] F. de Juan, A. G. Grushin, T. Morimoto, and J. E. Moore, Nat. Commun. 8, 15995 (2017).

[32] F. Flicker, F. de Juan, B. Bradlyn, T. Morimoto, M. G. Vergniory, and A. G. Grushin, Phys. Rev. B 98, 155145 (2018).

[33] G. Chang, S.-Y. Xu, B. J. Wieder, D. S. Sanchez, S.-M. Huang, I. Belopolski, T.-R. Chang, S. Zhang, A. Bansil, H. Lin, and M. Z. Hasan, Phys. Rev. Lett. 119, 206401 (2017).

[34] H. Nielsen and M. Ninomiya, Nucl. Phys. B 193, 173 (1981).

[35] H. Nielsen and M. Ninomiya, Nucl. Phys. B 185, 20 (1981).

[36] H. Nielsen and M. Ninomiya, Phys. Lett. B 105, 219 (1981).

[37] D. S. Sanchez, I. Belopolski, T. A. Cochran, X. Xu, J.-X. Yin, G. Chang, W. Xie, K. Manna, V. Sub, C.-Y. Huang, N. Alidoust, D. Multer, S. S. Zhang, N. Shumiya, X. Wang, G.-Q. Wang, T.-R. Chang, C. Felser, S.-Y. Xu, S. Jia, H. Lin, and M. Z. Hasan, Nature (London) 567, 500 (2019).

[38] P. Tang, Q. Zhou, and S.-C. Zhang, Phys. Rev. Lett. 119, 206402 (2017).

[39] D. Rees, K. Manna, B. Lu, T. Morimoto, H. Borrmann, C. Felser, J. E. Moore, D. H. Torchinsky, and J. Orenstein, Sci. Adv. 6, eaba0509 (2020).

[40] Z. Ni, B. Xu, M. A. Sanchez-Martinez, Y. Zhang, K. Manna, C. Bernhard, J. W. F. Venderbos, F. de Juan, C. Felser, A. G. Grushin, and L. Wu, arXiv:2005.13473.

[41] See Supplemental Material at http://link.aps.org/supplemental/ 10.1103/PhysRevB.102.121111 for derivation details of the symmetry analysis, which includes Refs. [30,31].

[42] J. R. Yates, X. Wang, D. Vanderbilt, and I. Souza, Phys. Rev. B 75, 195121 (2007).

[43] K. Koepernik and H. Eschrig, Phys. Rev. B 59, 1743 (1999).

[44] J. P. Perdew, K. Burke, and M. Ernzerhof, Phys. Rev. Lett. 77, 3865 (1996).

[45] J. Ibanez-Azpiroz, S. S. Tsirkin, and I. Souza, Phys. Rev. B 97, 245143 (2018).

[46] C. Wang, X. Liu, L. Kang, B.-L. Gu, Y. Xu, and W. Duan, Phys. Rev. B 96, 115147 (2017). 